\title{
Application of native prick test in diagnosis of bed bug allergy
}

\author{
Natalia Ukleja-Sokołowska, Łukasz Sokołowski, Ewa Gawrońska-Ukleja, Zbigniew Bartuzi
}

Department and Clinic of Allergy, Clinical Immunology and Internal Diseases, Nicolaus Copernicus University, Bydgoszcz, Poland Head: Prof. Zbigniew Bartuzi MD, PhD

Postep Derm Alergol 2013; XXX, 1: 62-64

DOI: 10.5114/pdia.2013.33382

\begin{abstract}
The aim of the study was case report of the patient with systemic reaction after a bed bug (Cimex lectularius) bite. A 23-year-old female, previously healthy, reports systemic reaction, including rash on her corpus and limbs, itching, nausea, conciseness disorder, forcing her to call the ambulance. The interview revealed that the bed bug occurs in the patient's apartment. A prick-by-prick test with bed bug excretion was made. The skin test with native allergen was strongly positive (histamine $5 \mathrm{~mm} / 5 \mathrm{~mm}$, prick-by-prick $12 \mathrm{~mm} / 8 \mathrm{~mm}$ ). The prick-by-prick test was useful in objective confirmation of the source of symptoms.
\end{abstract}

Key words: skin test, prick by prick, bed bug, anaphylactic reaction.

\section{Introduction}

Diagnosis of allergic diseases is often difficult. The clinician should pay particular attention to the interview, especially the nature of symptoms, duration, circumstances of occurrence and exposure to certain external factors. It is important both for the doctor and the patient to objectively confirm or exclude the symptoms, what has an impact on further disease management $[1,2]$.

Prick-by-prick tests with native allergens are a variety of classic skin prick tests (SPT). Both the European Academy of Allergy and Clinical Immunology (EAACI) and Polish Society of Allergology (PTA) allow the use of native tests for the diagnosis of food allergy when it is suspected that the result of SPT is false negative $[3,4]$.

There are certain clinical situations in which the prick-byprick test enabled confirmation of allergy against allergens other than food. In 2000, Kofler et al. described a case of a patient in which the prick-by-prick test with a leaf of a fern allowed to diagnose the source of rhinitis symptoms [5].

In 2001, Munno et al. recognized allergy to yucca in a patient complaining of swelling of the eyelids and urticaria. He used the prick-by-prick test with native yucca leaves and flowers [6]. Prick-by-prick tests have numerous limitations, but there are cases where their use is relatively safe, inexpensive and allows to give the patient a quick diagnosis.

\section{Case report}

The patient, 23 years old, female, in September 2010 had recurring incidents of rash on the trunk and limbs, accompanied by generalized itching of the whole body, confusion, abdominal pain and nausea. Symptoms occurred at night or during early morning and were waking the patient from sleep.

Three times because of severe symptoms the patient was forced to call an ambulance that drove her to the Emergency, where after anti-allergic drugs, including adrenaline, were given, symptoms resolved (13.06.2009 - 3.00 a.m., 16.10.2009 - 5.00 a.m., 20.06.2010 - 6.20 a.m.).

A detailed community interview revealed that the patient lived with her mother in a flat and her next-door neighbor was abusing alcohol, her flat was neglected and from time to time from the neighbor's apartment single insects approached the patient's flat. Those insects were probably bed bugs. The patient was not sure whether she in fact was bitten by an insect, because the response was generalized and the documentation provided by the patient did not mention any traces of bites at the time of examination by a physician in the hospital emergency department.

In order to confirm or exclude that the observed symptoms are connected with the bug, a prick-by-prick test was performed with oral secretions of insect mouthparts,

Address for correspondence: Natalia Ukleja-Sokołowska MD, PhD, Department and Clinic of Allergy, Clinical Immunology and Internal Diseases, Nicolaus Copernicus University, 30c Cieplicka Str., 85-377 Bydgoszcz, Poland, phone: +48 6919739 69, e-mail: ukleja@10g.pl Received: 30.08.2012, accepted: 12.11.2012. 
that the patient had caught in her apartment. An insect brought to the clinic was identified as a bed bug - Cimex lectularius.

The test result was strongly positive (histamine $5 \mathrm{~mm} /$ $5 \mathrm{~mm}$ - elevation, $30 \mathrm{~mm} / 30 \mathrm{~mm}$ erythema, prick-by-prick test $12 \mathrm{~mm} / 8 \mathrm{~mm}$ wheal, 35/30 erythema). The patient was instructed about the likely cause of her symptoms and recommended disinfestation facilities. We also clarified that in case of further exposure to bugs (e.g. in hotels), the patient should carry an automatic syringe with adrenaline, and also systemic steroids and antihistamines.

After eliminating bed bugs from the environment, the symptoms have not repeated so far.

The prick-by-prick test with bed bug secretion allowed to objectively confirm the source of symptoms. They proved to be a rapid, effective and safe method by which the diagnosis was established. After disinfection of the premises where the patient resides, the symptoms recurred.

\section{Discussion}

Bed bug (Cimex lectularius) is an insect commonly found around the world. The Cimicide family, which includes the bed bug, includes 91 species that are obligatory parasites, who consume the blood of mammals and birds. Only two species - Cimex lectularius and Cimex hemipterus - feed on human blood, but others may do so occasionally [7, 8].

The bug body is oval, strongly flattened, tapering towards the front, in red-brown color. Adult specimens reach the length of 4-5 mm, females are generally larger and rounder than males. The pentagonal head has a pair of antennae, and on the sides of the head there are large black eyes. Tube-like mouth-parts are well-adapted to piercing the skin and collecting the liquid food. Bugs body size increases after a meal by 30-50\%, and the weight increases by $150-200 \%[7,9]$.

Bed bugs in warm temperate climate can live up to 2 years. After fertilization, the female lays eggs, that depending on temperature, hatch after $4-6$ days (in $35-37^{\circ} \mathrm{C}$ ) or after $15-29$ days (in $14-16^{\circ} \mathrm{C}$ ). Lower temperatures inhibit the hatching of larvae. Over a lifetime the female may lay up to 500 eggs. The larvae pass incomplete development of the transformation [9].

Bed bugs avoid light, they parasitize during the night. During the day they hide in close proximity to the host, mostly in the mattresses of beds, headrests, behind paintings and bookshelves. Bed bugs can sense and turn to the heat, which allows them to locate the host. Stinging by a bug is painless, but after a few minutes there is a feeling of itching and burning. Insects can move from one apartment to another, also migrate between hotel rooms [7, 9].

Bed bugs are a lot more common in developing countries than in the developed ones. In the second half of the twentieth century, because of improvement of the sanitary conditions and introduction of effective insecticides, the population of these insects in Europe and North Amer- ica was reduced. In recent years, due to migration of people from developing countries, there has been a growth of parasitic infestation in countries such as Germany, the USA, Canada, Italy and Australia [10]. Bugs can "travel" with people on their clothes, luggage or furniture. It is called the passive spread [11].

Bed bugs bites in the majority of human population cause a slight, localized skin reaction, which disappears without complications within about a week. A large group of the population in general does not have a local reaction. Classically the affected area is circular, about 2-5 $\mathrm{mm}$ in diameter, red and slightly elevated above the level of the skin. Reactions often occur next to each other linearly. They are highly itchy and in their surroundings there are usually marks from scratching [12]. Skin reaction can occur several days after exposure to the bug [9]. Time after which the reaction occurs can be altered by multiple exposures. Usinger fed Cimex lectularius colony on his body in controlled conditions every week for 7 years and noticed that his skin reaction at the beginning was delayed and after several expositions - immediate [7].

Generalized reaction is rare and the descriptions are sporadic. Liebold et al. presented a patient who, after being bitten by the bug, suffered from a generalized bullous allergic reaction, which was accompanied by fever and general fatigue [13].

Scientists consider bugs as potential vectors of infectious diseases such as Q fever, Chagas disease, hepatitis B, HIV, MRSA and many others. In spite of this opinion, there is no conclusive evidence of transmission of infection by Cimex lectularius. The most advanced are researches on $\mathrm{HBV}$. In laboratory studies the presence of HBV in the body of bugs was detected 2 months after ingestion of infected blood. However, studies have not confirmed the possibility of viral replication or transferring the virus into a chimpanzee's body. In Gambia, no relation was noted between the eradication of insects in the rooms where children lived and the incidence of HBV infection, although the infection itself was very effective in eliminating bugs $[9,11]$.

Similarly, the possibility has not been demonstrated in HIV replication, or its transmission, although the virus was able to survive 8 days in the body of the bug [9].

Allergy to a bug is relatively rare, what is making its diagnosis difficult. Given the need for objective confirmation of allergies, in the case of our patient we decided to perform a skin test with native secretions of an insect mouthparts. Unfortunately, we failed to order a standardized allergen extract, because it was not available in Europe $[14,15]$. Moreover, in Poland it is impossible to determine allergen-specific IgE against bug allergens. The same problems and limitations are faced by researchers in Europe and the USA.

An interesting report on the diagnosis of allergy to bed bug was given by Prince et al. in a letter to the editorial board of the Journal of Allergy and Clinical Immunology, 
published in January 2012. The authors wanted to determine the specific lgE in patients with a history of a bed bug bite. It was observed that saliva contains bugs CNP - nitric oxide carrying protein. The unique structure of this protein suggests that increased concentrations of IgE specific for CNP is equal with hypersensitivity to the bug. The authors prepared an allergen extract from $160 \mathrm{mg}$ of dried Cimex lectularius. They also prepared a recombinant CNP protein from Escherichia coli. Authors assessed CNP-specific lgE against bed bug allergens in serum using the standard Immuno CAP method. They diagnosed 30 New York citizens who were bitten by an insect in the last year and reported local symptoms. Seventeen subjects had detectable IgE levels $\geq 0.1 \mathrm{lU} / \mathrm{ml}$ against the bed bug extract, and of these 9 had detectable levels of IgE against CNP. All patients with detectable lgE levels against CNP, had a detectable level of slgE against Cimex lectularius. The authors were unable to objectively assess the relation between the presence of specific lgE and the severity of symptoms due to the retrospective nature of the research and a small group of patients. Determination of specific IgE in the absence of specific standardized antigens must be regarded as a success $[16,17]$.

\section{Conclusions}

Domestic bed bug, usually associated with developing countries, increasingly appears in Western Europe. Keep it in mind as a potential source of symptoms in patients. Prick-by-prick tests with the secretions of bugs mouthparts allowed to objectively confirm the source of an anaphylactic reaction in our patient. Native prick tests, despite their limitations, are a relatively safe, fast and effective method.

\section{References}

1. Żukiewicz-Sobczak W, Krasowska E, Zwoliński J, et al. Allergic diseases - current state of knowledge. Postep Derm Alergol 2012; 29: 451-5.

2. Hallas TE, Gislason T, Gislason D. Mite allergy and mite exposure in Iceland. Ann Agric Environ Med 2011; 18: 13-7.

3. Kruszewski J, Kowalski M. Standards in allegology. Part I. Medycyna Praktyczna, Krakow 2010.

4. Stankiewicz-Choroszucha BL, Wawrzyniak ZM, Lipiec A, et al. Consequences of smoke inhalation in the 'Epidemiology of Allergic Diseases in Poland' project (ECAP). Ann Agric Environ Med 2011; 18: 420-8.

5. Kofler H, Hemmer W, Focke M, et al. Fern allergy. Allergy 2000; 55: 299-300.

6. Munno G, Giannoccaro F, Riva G, et al. Allergy to yucca. Allergy 2001; 56: 921

7. Usinger RL. Monography of cimicide. Vol 7. College Park, MD: Thomas Say Fundation 1966.

8. Leverkus M, Jochim RC, Schäd S, et al. Bullous allergic hypersensitivity to bed bug bites mediated by IgE against salivary nitrophorin. J Invastig Dermatol 2006; 126: 91-6.

9. Goddard J, De Shazo R. Bed bugs (Cimex lectularius) and clinical consequences of their bites. JAMA 2009; 301: 1358-66
10. Lee IY, Ree HI, An SJ. Reemergence of the bed bug Cimex lectularius in Seoul. Korean J Parasitol 2008; 46: 269-71.

11. Delaunay P, Blanc V, Del Giudice P. Bed bugs and infectious diseases. Clin Infect Dis 2011; 52: 200-10.

12. Massetti M, Bruschi F. Bed bugs infestation recorded in Central Italy. Parasitol Int 2007; 56: 81-3.

13. Liebold K, Schliemann-Willers S, Wollina U. Disseminated bullous eruption with systemic reaction caused by Cimex lectularius. J Eur Acad Dermatol Venereol 2003; 17: 461-3.

14. Allergy products catalogue. September 2008, www.allergopharma-nexter.pl.

15. Available at: http://www.stallergenes.com.

16. Price J, Divjanb A, Montfort W, et al. IgE against bed bug (Cimex lectularius) allergens is common among adults bitten by bed bugs. J Allergy Clin Immunol 2012; 129 (3 Suppl): 863-5.e1.

17. Available at: http://www.phadia.com/en/Allergen-information/ImmunoCAP-Allergens/. 